\title{
Cell culture-based analysis of postsynaptic membrane assembly in muscle cells
}

\author{
Getu Teressa ${ }^{1,2}$ and Joav Prives ${ }^{1 \sharp}$
}

\author{
${ }^{1}$ Department of Pharmacological Sciences, Stony Brook University, Stony Brook, NY 11794, USA \\ 2 Medical Scientist Training Program, Stony Brook University, Stony Brook, NY 11794, USA \\ \#Corresponding Author: Joav Prives, Ph.D. Department of Pharmacological Sciences, Stony Brook University, Stony Brook, NY $11794-8651$ \\ Phone: (631) 444 3139; Fax: (631) 444 3218; E-mail: joav@pharm.stonybrook.edu
}

Submitted: December 19, 2007; Revised: March 28, 2008; Accepted: April 1, 2008

Indexing terms: Acetylcholine receptors; Muscle; Agrin; Laminin; Synapse

Abbreviations: AChR, acetylcholine receptor; EGFP, enhanced green fluorescent protein; NMJ, neuromuscular junction

\begin{abstract}
We report a method for studying postsynaptic membrane assembly utilizing the replating of aneural cultures of differentiated skeletal muscle cells onto laminin-coated surfaces. A significant limitation to the current cell culturebased approaches has been their inability to recapitulate the multistage surface acetylcholine receptor (AChR) redistribution events that produce complex AChR clusters found at the intact neuromuscular junction (NMJ). By taking advantage of the ability of substrate laminin to induce advanced maturation of AChR aggregates on the surface of myotubes, we have developed a secondary-plating method that allows more precise analysis of the signaling events connecting substrate laminin stimulation to complex AChR cluster formation. We validate the utility of this method for biochemical and microscopy studies by demonstrating the roles of RhoGTPases in substrate laminin-induced complex cluster assembly.
\end{abstract}

\section{INTRODUCTION}

Prior to the innervation of embryonic skeletal muscle cells by motor neurons, surface nicotinic acetylcholine receptors (AChRs) are diffusely distributed on the surface of myotubes. Upon formation of nerve-muscle contacts, however, these AChRs aggregate into high-density patches in regions of the membrane directly underlying the motor nerve ending. The extracellular matrix (ECM) proteoglycan agrin secreted by motor neurons has been shown to be the major molecular trigger responsible for the aggregation of $\mathrm{AChR}$ and other synaptic components (1-4). In addition to agrin, some isoforms of the ECM glycoprotein laminin cause clustering of $\mathrm{AChR}$ on cultured muscle cells $(5,6)$, and appear to be crucial for normal development of the neuromuscular junction (NMJ) $(6,7)$. The initial stage of AChR clustering at developing NMJs in intact embryos involves the formation of ovoid aggregates (3), and can be mimicked in cell culture by applying a soluble recombinant form of neural agrin to myotubes $(8,9)$. Likewise, soluble laminin induces ovoid AChR clusters on cultured muscle cells $(6,7)$. However, nerve-induced clustering is significantly more elaborate; passing through several subsequent stages to form a region of elevated $\mathrm{AChR}$ density that is markedly more complex in structure (3). Consequently, to date, muscle cultures have been invaluable for the elucidation of the initial events in the formation of neuromuscular synapses, but far less useful for unraveling the sequence of subsequent steps crucial for the maturation of these structurally complex synapses.

Recently, it was shown that myotubes differentiated on substrate laminin display on their adherent surfaces an AChR aggregation process that more closely resembles postsynaptic specialization induced by innervation at NMJs (10). In order to determine the molecular mechanisms by which substrate laminin produces these complex AChR clusters, it is important to distinguish the direct focal actions of extracellular laminin at sites of 
cluster formation from the generalized stimulation by substrate laminin of the muscle differentiation process, since immobilized laminin has previously been shown to enhance skeletal myogenesis in vitro (11). Thus, the appearance of complex AChR clusters on these myotubes might reflect an indirect effect of the more extensive differentiation process. The secondary plating procedure that we describe in the present study makes it possible to bypass the effects of substrate laminin on muscle differentiation and to focus on the possibility that contact with immobilized laminin directly induces cluster formation and maturation. The current study successfully shows that immobilized laminin directly triggers $A C h R$ clustering pathways.

Moreover, the secondary plating method provides a means to determine the signaling events connecting laminin stimulation to complex AChR cluster formation. We have shown the utility of this approach by demonstrating the role of RhoGTPases in the coupling of immobilized laminin-derived signaling to the multistage AChR clustering process. The experiments described below reveal crucial roles for Rac and Rho that extend beyond those elucidated in oval-shaped cluster assembly by soluble forms of laminin and agrin (12-14). Moreover, we show that signals initiated by soluble recombinant neural agrin markedly modify the complex cluster inducing signals triggered by immobilized laminin, as revealed by the regression of mature clusters into ovoid clusters upon stimulation by agrin. Together, these findings show that this method is uniquely suitable for elucidating the molecular mechanisms coupling transmembrane signaling to the assembly of complex AChR-rich postsynaptic membrane in vitro.

\section{MATERIALS AND METHODS}

\section{Reagents}

Expression plasmids encoding dominant negative EGFPtagged Rho (GFP-RhoN19) were generously provided by Dr. M. Frohman and Dr. G. Du (Stony Brook University, Stony Brook, NY). A plasmid encoding GST fused to the Cdc42/Rac (p-21)-binding domain (GST-PBD) was a gift from Dr. J. Brugge (Harvard University, Boston, MA). The p160 ROCK inhibitor Y-27632 was purchased from Calbiochem. Laminin-1 was purchased from Invitrogen. A peptide encoding the c-terminal half of agrin was purchased from R \& D Systems. Other reagents were

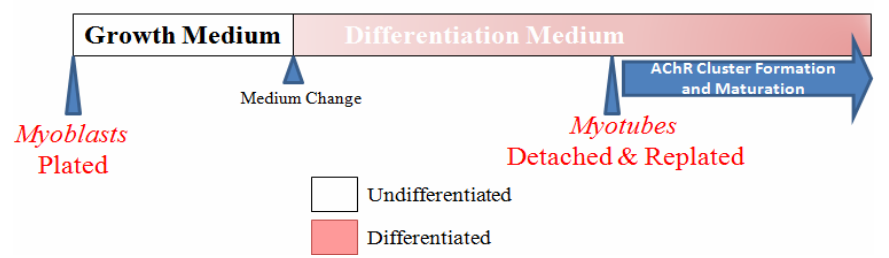

Fig. 1: Schematic representation of the secondary-plating method. C2C12 myoblasts were plated on uncoated plastic culture dishes in growth medium, and after two days, the medium was replaced by Differentiation Medium to induce myoblast fusion. Where indicated, myoblasts were transfected a day after plating. After 3 or 4 days in Differentiation Medium, myotubes were detached by mild trypsinization and replated onto laminin-coated $100 \mathrm{~mm}$ dishes for biochemical assays, or $12 \mathrm{~mm}$ laminin-coated coverslips for microscopy.

obtained from Sigma-Aldrich, unless otherwise stated.

\section{Cell culture and transfection}

C2 mouse myoblasts were plated on $100 \mathrm{~mm}$ culture dishes in Growth Medium consisting of Dulbecco's modified Eagle's medium (DMEM) supplemented with $10 \%$ fetal bovine serum, $5 \%$ calf serum and $100 \mu \mathrm{g} / \mathrm{ml}$ penicillin-streptomycin $37^{\circ} \mathrm{C}$ with air $/ 5 \% \quad \mathrm{CO}_{2}$. To stimulate muscle differentiation, two days after plating Growth Medium was replaced with Differentiation Medium consisting of DMEM containing 2\% horse serum and $100 \mu \mathrm{g} / \mathrm{ml}$ penicillin-streptomycin. Under these conditions the majority of $\mathrm{C} 2$ myoblasts fused to form multinucleated myotubes during the subsequent 3 days. Differentiated C2 myotubes were then replated onto $12 \mathrm{~mm}$ glass coverslips coated with laminin for microscopy, or onto other $100 \mathrm{~mm}$ culture dishes coated with laminin for biochemical assays. Coated surfaces were prepared by coating with $2 \mu \mathrm{g} / \mathrm{ml}$ polyornithine in sterile distilled water for 30 minutes and air dried for 30 minutes. Next, a $100 \mu \mathrm{g} / \mathrm{ml}$ solution of laminin in DMEM and/or a $50 \mathrm{ng} / \mathrm{ml}$ solution of agrin in DMEM was applied to the surfaces for 3 hours at $37^{\circ} \mathrm{C}$, prior to replating myotubes over these surfaces. Where indicated, myotube cultures were exposed overnight to $20 \mu \mathrm{g} / \mathrm{ml}$ of soluble laminin or $10 \mathrm{ng} / \mathrm{ml}$ of soluble agrin. For experiments involving transfection, myoblasts cultured on $100 \mathrm{~mm}$ tissue culture dishes were transfected one day post-plating with GFPRhoN19 plasmid at a final concentration of $5 \mu \mathrm{g}$ of $\mathrm{DNA} / \mathrm{ml}$ using Lipofectamine reagent (Invitrogen). The transfection medium was replaced with Differentiation Medium for 2-3 days prior to replating the differentiated myotubes on substrate laminin. 


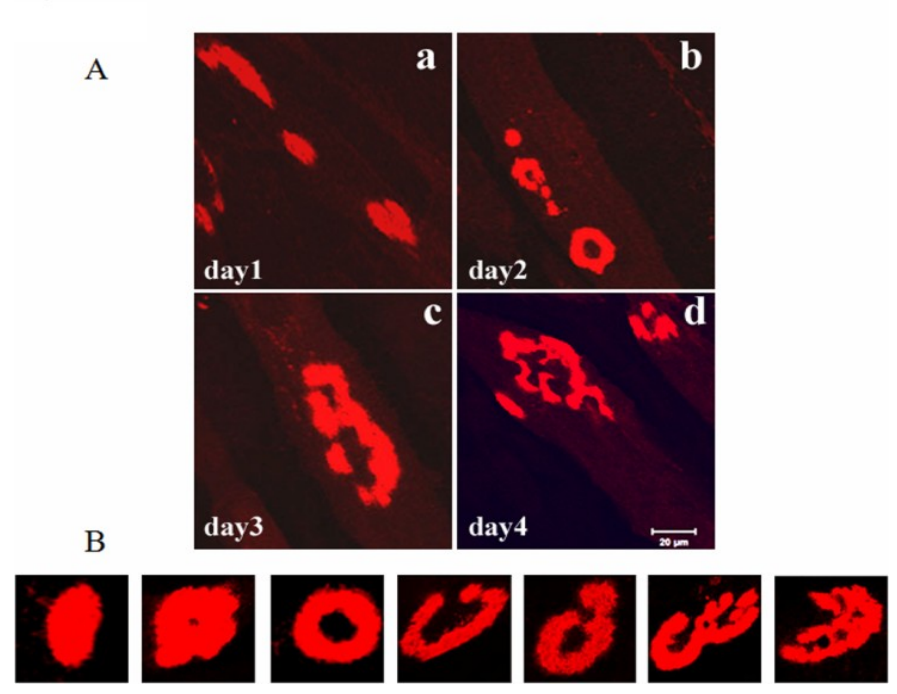

Fig. 2: Effects on $A C h R$ clustering of secondary plating of differentiated myotubes onto substrate-coated laminin. Differentiated myotubes replated on laminin-coated coverslips were seen to form increasingly complex and heterogeneous AChR clusters, as visualized by labeling AChRs on intact myotubes with TMR-Bgt (10nM for $1 \mathrm{~h}$ at $\left.37^{\circ} \mathrm{C}\right)$. The morphological maturation of AChR aggregates proceeds through the ovoid stage (2Aa; $2 \mathrm{~B})$ on day 1 to pretzel-like aggregates by day 4 postreplating (2Ad; 2B). Scale bar, 20 $\mu \mathrm{m}$

\section{Fluorescence microscopy}

To visualize surface distribution of AChR, cells were labeled with $10 \mathrm{nM}$ tetramethylrhodamine-conjugated $\alpha$ bungarotoxin (TMR-Bgt, Molecular Probes) in DMEM containing $1 \mathrm{mg} / \mathrm{ml}$ bovine serum albumin (BSA) for 1 hour at $37^{\circ} \mathrm{C}$, rinsed with Dulbecco's PBS and fixed in $3.7 \%$ formaldehyde-PBS for 30 minutes. Images were acquired using a Zeiss LSM510 confocal fluorescent microscope and associated imaging system with the 63X objective, and processed using Adobe Photoshop. AChR

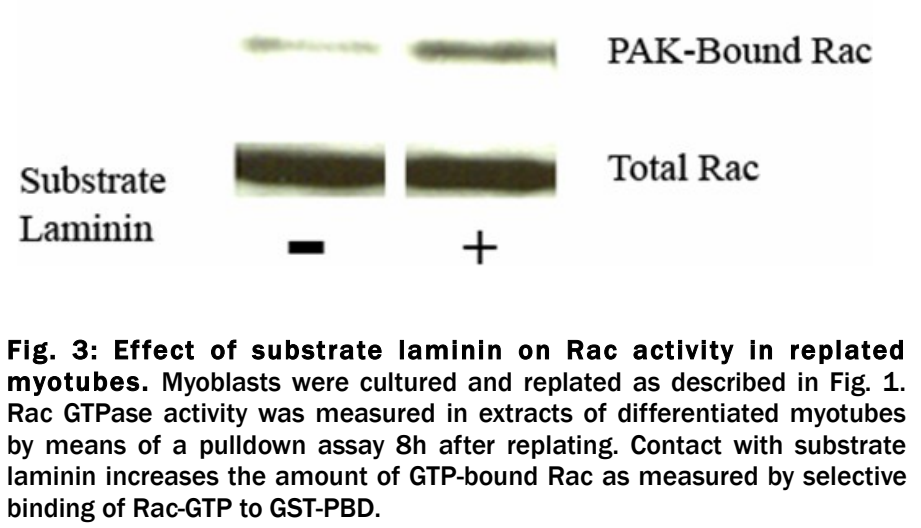
binding of Rac-GTP to GST-PBD. clusters were easily distinguished in TMR-Bgt-labeled cultures as discrete patches of intense fluorescence, and have characteristic appearances and dimensions, dependent on whether induced by soluble agrin or substrate laminin, as further described in the results. For quantitative determination, clusters in 50 randomlyselected myotubes in two separate experiments were viewed by fluorescence microscopy and counted.

\section{Rac1 Activity Assay}

Rac activation was measured by affinity precipitation of cellular GTP-bound forms of Rac (Sander et al., 1998). C2 myotubes replated onto immobilized laminin or polyornithine were rinsed with ice-cold Tris-buffered saline (TBS) supplemented with $1 \mathrm{mM} \mathrm{MgCl}_{2}$ and $0.5 \mathrm{mM} \mathrm{CaCl}_{2}$. Cells (one $100 \mathrm{~mm}$ plate per condition) were then lysed by incubation for 5 minutes on ice with lysis buffer $(50 \mathrm{mM}$ Tris- $\mathrm{HCl}, \mathrm{pH} 7.4,10 \mathrm{mM} \mathrm{MgCl} 2,10 \%$ glycerol, $100 \mathrm{mM}$ $\mathrm{NaCl}, 1 \mathrm{mM}$ benzamidine, $1 \mu \mathrm{g} / \mathrm{ml}$ leupeptin, $1 \mu \mathrm{g} / \mathrm{ml}$ pepstatin, $1 \mu \mathrm{g} / \mathrm{ml}$ aprotinin, $0.5 \%$ sodium deoxycholate), were centrifuged for 5 minutes at $11,000 \mathrm{rpm}$ at $4{ }^{\circ} \mathrm{C}$, and the supernatants were used as cell lysates and incubated with GST fused to the Cdc42/Rac (p-21)-binding domain of PAK bound to glutathione-coupled sepharose beads (GST-PBD) for 30 minutes at $4^{\circ} \mathrm{C}$. The fusion protein beads with bound proteins were then washed three times with wash buffer $(50 \mathrm{mM}$ Tris, $\mathrm{pH} 7.2$, containing $1 \%$ Triton $X-100,150 \mathrm{mM} \mathrm{NaCl}, 10 \mathrm{mM} \mathrm{MgCl}_{2}, 10 \mu \mathrm{g} / \mathrm{ml}$ each of leupeptin and aprotinin, and 0.1mM PMSF), eluted in sample buffer and analyzed by western blotting with a mouse monoclonal antibody against Rac1 (Transduction Labs). Blots were developed using sheep anti-mouse antibody coupled to horseradish peroxidase (HRP) and visualized with the Western Lighting Chemiluminescence Reagent (PerkinElmer, Inc.).

\section{RESULTS}

\section{Secondary plating of myotubes onto immobilized laminin to induce complex AChR clusters}

Soluble laminin is known to induce ovoid-shaped AChR clusters on muscle cells $(5,6)$ reminiscent of early stages of postsynaptic membrane differentiation upon innervation (3). In contrast, cultured muscle cells plated and differentiated in contact with immobilized laminin display 

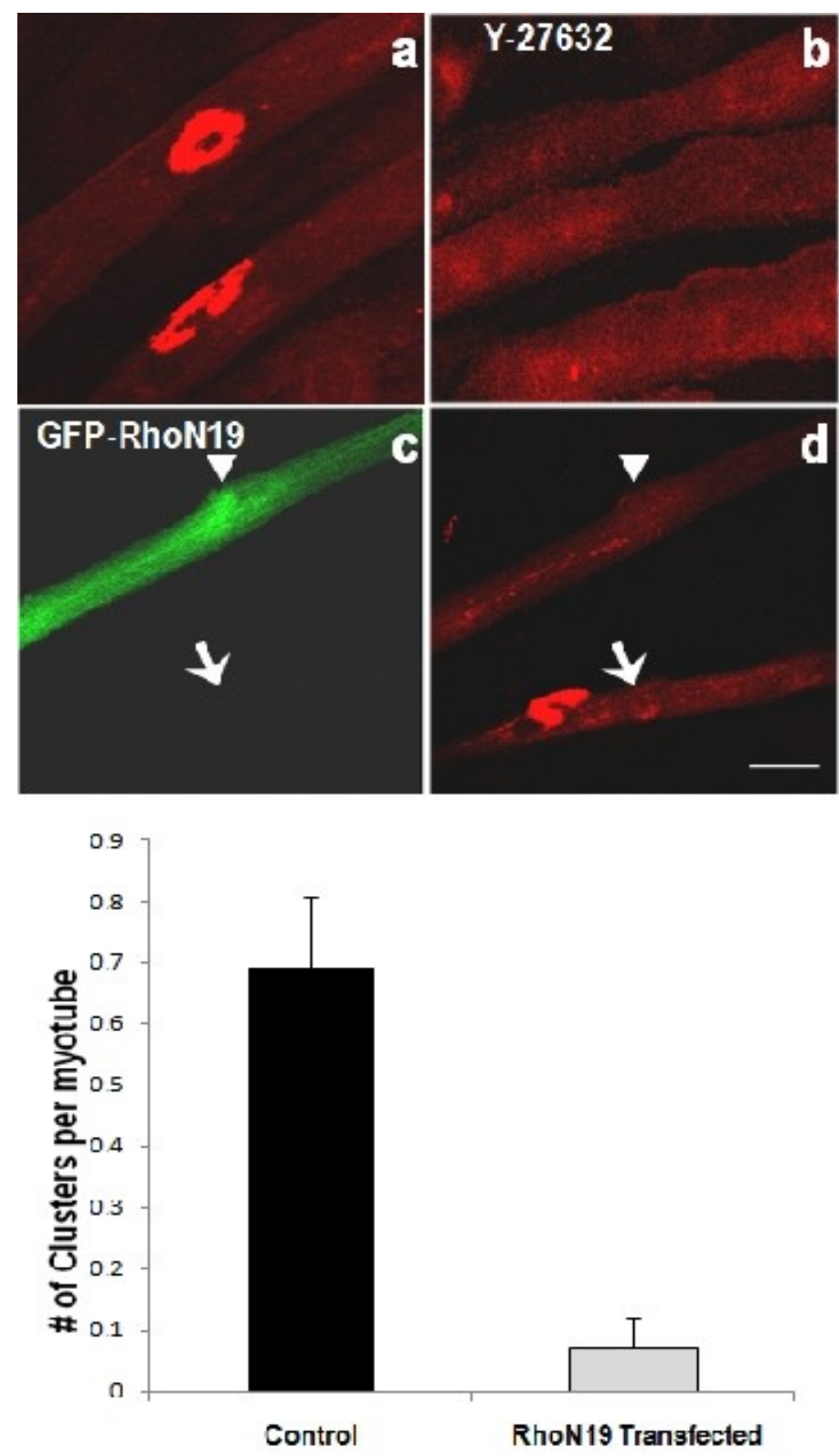

Fig. 4: Effects of blocking Rho-mediated signaling on AChR clustering induced by substrate laminin. Confocal microscopy of replated cultures labeled with TMR-Bgt was conducted to visualize the induction by substrate laminin of complex clusters (Fig. 4A panel a). Inhibition of the Rho effector, ROCK, using a pharmacological inhibitor Y-27632, is seen to block assembly of complex AChR clusters (Fig. 4A panel b). Expression of a dominant negative Rho mutant (GFP-RhoN19) in muscle cells (Fig. 4A panel c, arrowhead) can be seen to block the formation of AChR clusters after replating onto immobilized laminin (Fig.4A panel d, arrowhead) compared to non-transfected myotubes in the same field (Fig. 4A panels $c, d$ arrow). Fig. 4B is a quantitative comparison of substrate laminin-induced clusters of various morphologies in these RhoN19 expressing myotubes versus untransfected cells. The total number of clusters is significantly reduced in RhoN19 expressing myotubes. Scale bar $20 \mu \mathrm{m}$. $(n=100$, Error bars represent $+/$-SEM).

far more complex clusters of cell surface AChR resembling mature postsynaptic membranes of innervated muscle cells (10). We first wanted to use the secondary plating method to determine if acute contact with substrate laminin can induce similarly complex AChR clusters on myotubes. In this method, cultures are allowed to differentiate into multinucleated myotubes on culture dishes in the absence of exogenous laminin substrate. The newly fused myotubes, which express AChR that is diffusely distributed on the cell surface, are then detached by mild trypsinization and replated onto laminin-coated coverglasses (Fig. 1). Upon contact with the laminincoated substrate, ovoid AChR clusters appeared on the underside of reattached myotubes within 8-12 hours, as an initial stage of AChR aggregation (Fig. 2Aa, B). Subsequently, cluster morphology continued to increase in complexity (Fig. 2) until elaborate pretzel-shaped AChR aggregation structures reminiscent of postsynaptic regions of innervated muscle were achieved 2-3 days after ovoid clusters first appeared (Fig. 2Ad, B).

\section{Biochemical measurements on replated myotubes}

The replating method can be exploited to investigate the early molecular events triggered by contact of fully differentiated myotubes to substrate laminin as well as the subsequent signaling events that regulate the maturation of AChR clusters. We have previously shown that activation of Rac1, a small GTPase that regulates actin polymerization and plays crucial roles in cell membrane dynamics (15-18), is critical for AChR aggregation into ovoid clusters in the presence of soluble agrin or laminin (12-14). Using the replating method, this time onto coated $100 \mathrm{~mm}$ culture dishes, we observed that cellular Rac1 is activated within $8 \mathrm{~h}$ of plating, before the appearance of AChR complex clusters on the adherent muscle cell surface (Fig. 3), demonstrating the utility of this method for biochemical signaling assays associated with AChR aggregation by immobilized ECM components.

\section{Use of fluorescence microscopy to analyze signaling mechanisms in replated myotubes}

To assess the contribution of the Rho effector pathway to AChR complex cluster formation triggered in myotubes by replating onto laminin-coated surfaces, we tested the effects on this process of a dominant negative mutant of Rho, RhoN19, as well as Y-27632, a pharmacological inhibitor of the Rho effector p160ROCK $(19,20)$. As shown 

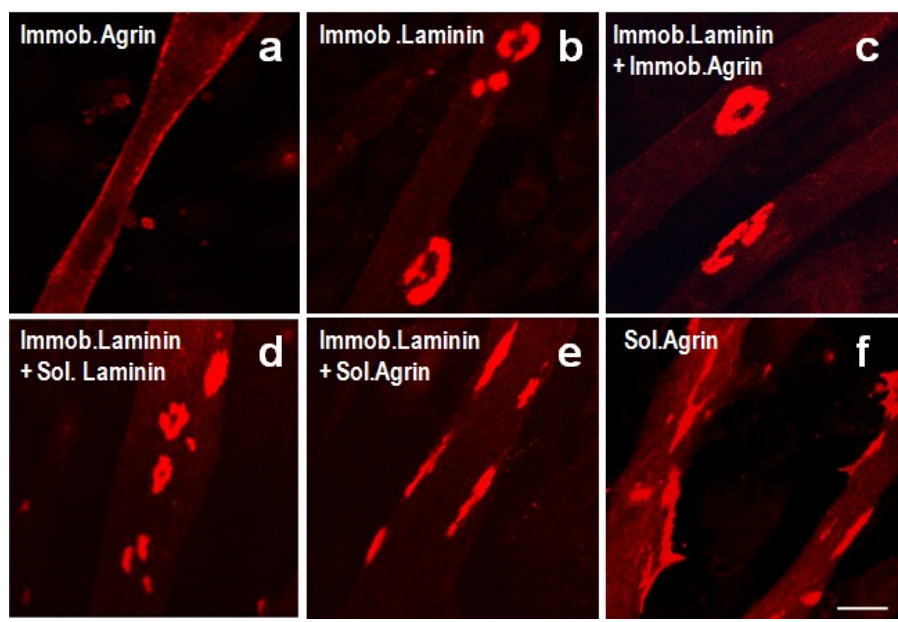

Fig. 5: Combinatorial effects of agrin and laminin in replated myotubes. To study the combined effects of agrin and laminin, replated myotubes were used to analyze AChR aggregation patterns induced by soluble and immobilized agrin and laminin. AChR clusters on myotubes replated onto substrate coated with laminin are shown in panel b. Agrin has no effect when immobilized either alone (panel a) or in combination with substrate laminin (panel $c$ ), while soluble agrin redistributes substrate laminin-induced complex AChR aggregation (panel e) into a pattern more characteristic of soluble agrin (panel f). Unlike soluble agrin, soluble laminin does not have any discernible effect on substrate laminin induced complex AChR cluster formation (panel d). Scale bar, $20 \mu \mathrm{m}$. Immob=Immobilized; Sol=Soluble.

in Fig. 4A, RhoN19-expressing myotubes replated onto laminin displayed a drastically reduced number of clusters as compared to controls, measured 3 days after replating. Addition of Y-27632 $(20 \mu \mathrm{M}$, added immediately upon replating and subsequently re-added every $8 \mathrm{~h}$ ) was seen to block the aggregation of $A C h R$ induced by immobilized laminin at 3 days after replating. Figure $4 \mathrm{~B}$ is a quantitative measurement verifying the inhibition by RhoN19 of complex cluster formation. These findings confirm the ability of the secondary plating method to define the role of Rho GTPases in the events that couple laminin contact to $\mathrm{AChR}$ cluster formation.

\section{Measurement of combinatorial effects of ECM components in replated myotubes}

We have utilized the myotube replating protocol to study the combined actions of soluble and immobilized forms of agrin and laminin on AChR aggregation. We found that soluble agrin radically modifies the aggregation pattern of AChR that is induced by immobilized laminin. Exposure to soluble agrin reorganizes the complex clusters into a simpler, ovoid morphology characteristic of agrin clusters as well as their subsequent displacement to the myotube periphery by 2 days after the initial exposure to agrin (Fig 5e). Surprisingly, these effects are manifested even Teressa and Prives - Cell culture-based analysis of postsynaptic membrane assembly in muscle cells www.biologicalprocedures.com under conditions where the exposure duration of replated myotubes to agrin is as brief as $15 \mathrm{~min}$.

\section{DISCUSSION}

The replating of differentiated $\mathrm{C} 2 \mathrm{C} 12$ myotubes onto immobilized laminin induces the aggregation of $\mathrm{AChR}$ into clusters that are highly extensive, and more reminiscent of the situation in vivo than is achievable with soluble laminin or agrin. Whereas the AChR aggregation state achieved by these soluble factors is limited to the formation of ovoid AChR clusters - an early stage in the overall postsynaptic differentiation process - the large AChR aggregates induced by substrate laminin closely resemble those found in the subsynaptic membranes of innervated muscle cells. Consequently this procedure allows a more complete characterization of the molecular mechanisms that direct aggregation of surface $A C h R$ into complex clusters.

The secondary plating approach derives from the interesting recent finding that myoblasts grown on substrate laminin differentiate into myotubes that display morphologically complex AChR clusters(10). Immobilized laminin, but not soluble laminin, has been shown to enhance general skeletal muscle differentiation in vitro (11) and thus it appeared possible that the complexity of AChR clusters reflects the extent of muscle differentiation. When myoblasts differentiated in the absence of exogenous laminin were subsequently replated onto laminin-coated substrate, they formed complex clusters indistinguishable from those observed on cells grown on laminin. This shows that this clustering response is due to the direct inductive role of immobilized laminin rather than reflecting an indirect effect of the enhancement of muscle differentiation by substrate laminin. Thus acute contact with immobilized laminin is sufficient to induce complex clustering.

This experimental technique can be used to study the signaling pathways by which contact with immobilized laminin triggers multistage AChR cluster assembly. In order to validate this approach, we have investigated the roles of Rac1 and RhoA in coupling substrate laminin signaling to the formation of branched $\mathrm{AChR}$ aggregates. It was previously shown that these Rho GTPases have complementary actions in mediating the formation of ovoid AChR clusters induced by soluble laminin and agrin $(12,14)$. We observed that acute contact of myotube 
membranes to substrate laminin induces the activation of Medical Sciences (NIGMS).

Rac1 prior to the appearance of complex clusters on the adherent surfaces of myotubes (Fig. 3). In addition, the dominant negative Rho mutant RhoN19 as well as the Rho kinase inhibitor Y-27632 block cluster formation on myotubes replated onto immobilized laminin (Fig. 4).

In muscle cultures, agrin and laminin induce AChR clustering via the activation of converging pathways (21-23) and have been shown to have synergistic roles in elevating the number of ovoid clusters on cultured myotube surfaces (22). Using the replating approach, we have now observed a surprising antagonistic effect of inputs from agrin and immobilized laminin. Our findings show that complex $\mathrm{AChR}$ aggregates assembled on myotube surfaces replated onto immobilized laminin regress into simple ovoid aggregates after even brief (15min) stimulation by soluble C-terminal agrin. We have also observed that these ovoid clusters are subsequently displaced from the under-surface to the edge of muscle cells during the next $24 \mathrm{~h}$.

In conclusion, the replating of myotubes grown on uncoated dishes onto surfaces coated with laminin has been shown here to have multiple applications in the elucidation of the roles of immobilized ECM components and other signaling molecules in the formation of specialized AChR-rich membrane regions closely resembling postsynaptic membranes at the NMJ. This method has enabled us to analyze the contributions of Rac1 and RhoA in the assembly of morphologically complex AChR aggregates.

The replating approach should allow elucidation of the signaling pathways underlying the multistage process that produces elaborately branched $\mathrm{AChR}$ aggregates that approximate in complexity the motor end-plate of innervated muscle. Because in the case of soluble laminin and agrin the clustering process is arrested at the ovoid cluster stage, the subsequent differentiation of high density AChR membrane regions has not heretofore been accessible to in vitro studies of this type.

\section{ACKNOWLEDGMENTS}

We thank Dr. Christi Weston for critical discussions. GT was supported by a Medical Scientist Training Grant and the Ruth L. Kirschstein National Research Service Award GM78814-01 from the National Institute of General

\section{REFERENCES}

1. Glass DJ et al. Agrin acts via a MuSK receptor complex. Cell 1996; 85:513-23.

2. Hoch W. Formation of the neuromuscular junction. Agrin and its unusual receptors. Eur J Biochem 1999; 265:1-10.

3. Sanes JR, Lichtman JW. Development of the vertebrate neuromuscular junction. Annu Rev Neurosci 1999; 22:389-442.

4. Sanes JR, Lichtman JW. Induction, assembly, maturation and maintenance of a postsynaptic apparatus. Nat Rev Neurosci 2001; 2:791-805.

5. Patton BL et al. Properly formed but improperly localized synaptic specializations in the absence of laminin alpha4. Nat Neurosci 2001; 4:597-604.

6. Patton BL, Miner JH, Chiu AY, Sanes JR. Distribution and function of laminins in the neuromuscular system of developing, adult, and mutant mice. J Cell Biol 1997; 139:1507-21.

7. Sugiyama JE, Glass DJ, Yancopoulos GD, Hall ZW. Laminin-induced acetylcholine receptor clustering: an alternative pathway. J Cell Biol 1997; 139:181-91.

8. Burden SJ. The formation of neuromuscular synapses. Genes Dev 1998; 12:133-48.

9. Colledge $M$, Froehner SC. To muster a cluster: anchoring neurotransmitter receptors at synapses. Proc Natl Acad Sci U S A 1998; 95:3341-3.

10. Kummer TT, Misgeld T, Lichtman JW, Sanes JR. Nerveindependent formation of a topologically complex postsynaptic apparatus. J Cell Biol 2004; 164:1077-87.

11. Foster RF, Thompson JM, Kaufman SJ. A laminin substrate promotes myogenesis in rat skeletal muscle cultures: analysis of replication and development using antidesmin and anti-BrdUrd monoclonal antibodies. Dev Biol 1987; 122:11-20.

12. Weston C, Gordon C, Teressa G, Hod E, Ren XD, Prives J. Cooperative regulation by Rac and Rho of agrininduced acetylcholine receptor clustering in muscle cells. J Biol Chem 2003; 278:6450-5.

13. Weston C, Yee B, Hod E, Prives J. Agrin-induced acetylcholine receptor clustering is mediated by the small guanosine triphosphatases Rac and Cdc42. J Cell Biol 2000; 150:205-12.

14. Weston CA, Teressa G, Weeks BS, Prives J. Agrin and laminin induce acetylcholine receptor clustering by convergent, Rho GTPase-dependent signaling 
pathways. J Cell Sci 2007; 120:868-75.

15. Bishop AL, Hall A. Rho GTPases and their effector proteins. Biochem J 2000; 348:241-55.

16. Hall A. Rho GTPases and the actin cytoskeleton. Science 1998; 279:509-14.

17. Ridley AJ. Rho family proteins: coordinating cell responses. Trends Cell Biol 2001; 11:471-7.

18. Takai Y, Sasaki T, Matozaki T. Small GTP-binding proteins. Physiol Rev 2001; 81:153-208.

19. Ishizaki $\mathrm{T}$ et al. Pharmacological properties of Y-27632, a specific inhibitor of rho-associated kinases. Mol Pharmacol 2000; 57:976-83.

20. Uehata $\mathrm{M}$ et al. Calcium sensitization of smooth muscle mediated by a Rho-associated protein kinase in hypertension. Nature 1997; 389:990-4.

21. Burkin DJ, Gu M, Hodges BL, Campanelli JT, Kaufman SJ. A functional role for specific spliced variants of the alpha7beta1 integrin in acetylcholine receptor clustering. J Cell Biol 1998; 143:1067-75.

22. Lee LK, Kunkel DD, Stollberg J. Mechanistic distinctions between agrin and laminin-1 induced aggregation of acetylcholine receptors. BMC Neurosci 2002; 3:10.

23. Montanaro $\mathrm{F}$ et al. Laminin and alpha-dystroglycan mediate acetylcholine receptor aggregation via a MuSK-independent pathway. J Neurosci 1998; 18:1250-60.

\section{PROTOCOL}

\section{Reagents}

- $\quad$ 22C12 cells

- Culture Media

Growth Medium: Dulbecco's modified Eagle's medium (DMEM) supplemented with $10 \%$ fetal bovine serum, $5 \%$ calf serum and $100 \mu \mathrm{g} / \mathrm{ml}$ penicillin-streptomycin.

Differentiation Medium: DMEM containing $2 \%$ horse serum and $100 \mu \mathrm{g} / \mathrm{ml}$ penicillin-streptomycin

- Phosphate Buffered Saline (PBS)

- Trypsin-EDTA

- Transfection Reagent, Lipofectamine

- $\quad 35 \mathrm{~mm}$ and $100 \mathrm{~mm}$ tissue culture dishes

- $12 \mathrm{~mm}$ coverslips

- Poly-Ornithine

- Agrin

- Laminin

- Tetramethylrhodamine-conjugated $\alpha$--bungarotoxin (TMR-Bgt)

\section{Protocol}

\section{Cell Culture}

1. Plate C2 mouse myoblasts on 100mm culture dishes in Growth Medium consisting of Dulbecco's modified Eagle's medium (DMEM) supplemented with $10 \%$ fetal bovine serum, $5 \%$ calf serum and $100 \mu \mathrm{g} / \mathrm{ml}$ penicillinstreptomycin and incubate $37^{\circ} \mathrm{C}$ with air $/ 5 \% \mathrm{CO}_{2}$.

2. For experiments involving transfection, transfect myoblasts cultured on $100 \mathrm{~mm}$ tissue culture dishes one day post-plating with the desired plasmid at a final concentration of $5 \mu \mathrm{g}$ of DNA $/ \mathrm{ml}$.

3. Two days after plating, or a day after transfection, replace the Growth Medium with Differentiation Medium consisting of DMEM containing $2 \%$ horse serum and $100 \mu \mathrm{g} / \mathrm{ml}$ penicillin-streptomycin to stimulate muscle differentiation.

4. In the subsequent 3 or 4 days, the majority of $\mathrm{C} 2$ myoblasts fuse to form multinucleated myotubes. Change Differentiation Medium every other day. 


\section{Preparation of Laminin-Coated Surfaces}

1. After 3 or 4 days in Differentiation Medium, when the $C 2$ myotubes are fully differentiated, prepare $12 \mathrm{~mm}$ glass coverslips coated with laminin for microscopy, or $100 \mathrm{~mm}$ culture dishes coated with laminin for biochemical assays.

2. Prepare laminin-coated surfaces by precoating first with $2 \mu \mathrm{g} / \mathrm{ml}$ polyornithine in sterile distilled water for 30 minutes and air dry for approximately $30 \mathrm{~min}$.

3. Incubate with $100 \mu \mathrm{g} / \mathrm{ml}$ solution of laminin in DMEM over polyornithine-coated dishes for 3 hours at $37^{\circ} \mathrm{C}$.

\section{Replating}

1. Detach the newly fused myotubes by mild trypsinization and replate them onto laminin-coated culture dishes or coverglasses.

2. To detach the cells, rinse the culture two times with sterile 1X PBS and incubate with 1ml 1XTrypsin-EDTA for 5-10 min.

3. Resuspend the myotubes gently in DMEM and pellet by centrifuging at 4000rpm for 10min.

4. Resuspend the myotube pellets gently with Differentiation Medium and transfer cells onto laminin coated surfaces. Surface distribution of AChR as well as biochemical measurements can be carried out at different time points after the myotubes start spreading and reattaching on the laminin substrate. 